\title{
Transoral Robotic Surgery (TORS) for the Head and Neck: Current and Future Indications
}

\author{
${ }^{1}$ Harry Quon, ${ }^{2}$ Bert W O'Malley (Jr), ${ }^{2}$ Gregory S Weinstein \\ ${ }^{1}$ Department of Radiation Oncology and Otorhinolaryngology, Head and Neck Surgery, Abramson Cancer Center, University of \\ Pennsylvania, Philadelphia, USA \\ ${ }^{2}$ Department of Otorhinolaryngology, Head and Neck Surgery, Abramson Cancer Center, University of Pennsylvania, Philadelphia, USA
}

Correspondence: Harry Quon, Hospital of the University of Pennsylvania, 3400 Spruce Street-2 Donner, Philadelphia, PA 19104, USA, Phone: 215-614-0392, Fax: 215-349-5445, e-mail: quon@xrt.upenn.edu

\begin{abstract}
Transoral robotic surgery (TORS) offers many technical advancements to existing endoscopic and transoral surgical approaches. This has faciliated a safer, less morbid and potentially more effective application of surgery to the management of both benign and malignant diseases in the head and neck. As this surgical approach gains widespread acceptance, it is important for all members of the treatment team to understand the strengths and current limitations especially when TORS is applied for malignant diseases. As of December 2009, Federal Drug Administration (FDA) has approved the use of the da Vinci ${ }^{\circledR}$ surgical system and TORS for selected malignancies of the oral cavity, pharynx and larynx and all benign disease. Of these sites, the greatest experience and longest duration of follow-up has been in the use of TORS for the management of oropharyngeal carcinomas where at least comparable oncologic outcomes and reduced long-term feeding tube dependency rates have been reported. Other anatomic sites where TORS has shown benefit based on preclinical studies and early human experiences include the larynx, hypopharynx, parapharyngeal space and infratemporal fossa for both benign and selected malignant tumors. Experience to date has demonstrated that the improved visualization with the robotic system offers the potential for improved oncologic resection with reduced morbidity. Based on present studies and outcomes data in conjunction with ongoing investigations, it is anticipated that TORS will make a major impact in the way we manage benign and malignant tumors within the head and neck and skull base.
\end{abstract}

Keywords: Transoral robotic surgery (TORS), head and neck neoplasms, skull base neoplasms, indications, postoperative radiotherapy, review.

\section{INTRODUCTION}

Transoral robotic surgery (TORS) represents a technologic evolution of transoral surgical approaches that is expanding the scope and current indications of surgical resection for many benign and malignant diseases in the head and neck. With growing interest and acceptance of TORS, it is important that the members of the treatment team understand the strengths and current limitations of TORS, especially when used for malignant diseases. As a result, we will review the current and evolving indications for TORS for the management of benign and malignant diseases in the head and neck (Table 1).

Table 1: Current indications for transoral robotic surgery

\begin{tabular}{|c|c|c|c|}
\hline Anatomic site & Preclinical experience & Clinical experience & Indications \\
\hline Oropharynx & Yes & Yes & $\begin{array}{l}\text { 1. Benign tumors } \\
\text { 2. Selected } \mathrm{T} 1-\mathrm{T} 2, \mathrm{~T} 3, \mathrm{~T} 4 \mathrm{a} \\
\text { oropharyngeal carcinomas }\end{array}$ \\
\hline Larynx/Hypopharynx & Yes & Yes & $\begin{array}{l}\text { 1. Benign tumors } \\
\text { 2. Selected T1, T2 and T3 } \\
\text { laryngeal and hypopharyngeal } \\
\text { carcinomas }\end{array}$ \\
\hline $\begin{array}{l}\text { Parapharyngeal space/ } \\
\text { Infratemporal fossa }\end{array}$ & Yes & Yes & 1. Benign tumors \\
\hline Anterior and middle skull base & Yes & No & \\
\hline Nasopharynx & Yes & Yes & $\begin{array}{l}\text { 1. Early experience in recurrent } \\
\text { T1-2 nasopharyngeal carcinoma }\end{array}$ \\
\hline
\end{tabular}




\section{STRENGTHS AND LIMITATIONS OF TRANSORAL ROBOTIC SURGERY (TORS)}

TORS has been the focus of significant preclinical and clinical studies establishing the issues of feasibility, safety and current indications. ${ }^{1-8}$ Ongoing studies suggest the potential for a scope of indications. ${ }^{9}$ TORS offers several technologic advancements to standard transoral and endoscopic surgical approaches that have been described to be effective. ${ }^{10}$ These include improved magnified threedimensional visualization allowing angled sight lines that facilitate two to four handed surgical resection, and a range of precise tremor-free wristed instruments that navigate through small openings and around anatomical structures. These properties allow fascile, safe, and efficient surgery without the surgical morbidity associated with open surgical exposure. As a result of the implementation of robotic technology, surgical complications and hospital admission time have been reduced. ${ }^{11}$

A key concern of new and minimally invasive surgical procedures is the issue of whether complete surgical resection is possible or not and whether there is an increased risk of close or positive margins versus classic large open surgical procedures. This concern raises question as to how confident one may be with recommending observation with no adjuvant therapy after achieving a TORS negative margin for malignancies, such as squamous cell carcinomas of the oropharynx. The answer to this question is not immediately available from the TORS experience that has been reported to date. At the University of Pennsylvania, the majority of patients with advanced oropharyngeal carcinomas enrolled in our study in its initial years received postoperative radiotherapy (PORT). As we have become more confident and the success of effective minimally invasive complete tumor resection with confirmed negative margins is achieved, we are planning to investigate the benefit and outcomes of eliminating adjuvant therapy. In considering the potential risks involved with the new paradigm, we have reviewed the published experience on the use of transoral laser microsurgery (TLM) for T1 and T2 oropharyngeal carcinomas.

Transoral resection of oropharyngeal carcinomas with the TLM technique has been reported by several groups. ${ }^{12-16}$ In contrast to the TORS technique, TLM uses a piecemeal resection technique until the tumor/normal tissue interface is reached. Sufficient safety margin is only confirmed by using the operative microscope to distinguish cancer from the normal tissues and resecting just beyond that interface. This approach along with the tissue artifacts reduced by laser can cast doubt on the confidence of the negative margin rate of this technique. A TORS approach obviates the concerns about surgical margins when compared to TLM since TORS allows for en bloc resection and a more confident margin assessment with whole specimen evaluation at the time of frozen and permanent section analysis. ${ }^{17}$ With a mean follow-up of 44 months, Grant et al have recently updated their experience with TLM alone for T1-3 disease demonstrating that the risk of local relapse was $90 \%$ and $94 \%$ for T1 and T2 disease respectively. ${ }^{14}$ While no local relapses were noted for T3 disease, there were only 12 of 69 patients in this subset. ${ }^{14}$

With transcervical neck approaches, Ang et al have demonstrated that the risk of local-regional relapse with no adverse pathologic risk factors is at least $10 \%{ }^{18}$ This risk reflects not only the false negative risk of a pathologic negative margin but also the risks associated with tumor seeding of the neck which in principle is reduced if not eliminated with TORS accompanied by a staged neck dissection.

As such, T1 and T2 disease with a negative TORS margin are likely associated with a $<10 \%$ risk of local relapse. Continued experience is needed to validate this statement. The TLM experience, however, does provide confidence that observation may be an appropriate treatment option following negative margins for $\mathrm{T} 1$ and $\mathrm{T} 2$ oropharyngeal carcinomas. It is important to note that $\mathrm{T}$ stage designation does not fully define the risk of residual disease but rather, it is important to consider the invasive nature and depth of the tumor. This is an intraoperative assessment necessitating clear communication between the surgeon and the radiation oncologist. Until further evidence accrues for a surgery alone approach for $\mathrm{T} 3$ tumors with a TORS negative margin, PORT should still be favored. However, exophytic lesions with a well-defined base and limited depth of tissue invasion may potentially be observed with no adjuvant therapy.

Traditional transcervical surgical approaches for oropharyngeal carcinomas typically resulted in significant morbidity with the risk of swallowing and speech dysfunction. While transoral surgical approaches obviate, these complications that are the results of surgical exposure, the issue of how much of normal base of tongue and pharyngeal muscle may be removed, before function is affected, has not been clearly established. ${ }^{19}$ Haughey and 
colleagues have demonstrated that with appropriate fasciocutaneous flap reconstruction, normal swallowing could be achieved with resection of up to $2 / 3$ of the base of tongue. ${ }^{19}$ Swallowing was assessed using the Functional Outcome Swallowing Scale (FOSS) developed by Salassa and colleagues. ${ }^{20}$ Such observations support the ability to take significant portions of the tongue base while retaining adequate speech and swallowing function. What remains unknown is the functional impact of resection of other pharyngeal structures involved in swallowing, such as the constrictor muscles and the epiglottis and how adjuvant therapy after TORS or TLM impacts the postsurgical swallowing and speech function objectively and its impact on patient quality of life. At the University of Pennsylvania, swallowing function assessed using the Performance Status Scale - Head and Neck instrument, was found not to be significantly different from baseline for eating and diet domains in 1 year. In 6 months, there was significant impairment to baseline that likely reflected the impairment due to postoperative radiotherapy (unpublished results).

At the University of Pennsylvania, general recommendations have been to limit TORS to situations where the resection does not involve the removal of more than $1 / 2$ of the base of tongue or resection of more than $1 / 2$ of the posterior pharyngeal wall. Even with aggressive TORS resection of OP tumors with postoperative adjuvant radiotherapy (and concurrent chemotherapy for positive margins and ECE), with a minimum 2 years follow-up, the percutaneous gastrostomy (PEG) tube dependency rate was $0 \%{ }^{21}$ These results suggest that this approach offers comparable if not lower risks of late swallowing complications. In a limited follow-up study of 12.5 months, Moore and colleagues also reported a 0\% PEG dependency rate with an identical policy of elective irradiation of the primary site despite negative margins after TORS in 45 patients. ${ }^{22}$ Comparable low rates of PEG dependency have also been observed by several other groups who have used other transoral nonrobotic surgical approaches with PEG dependency rates of $0 \%{ }^{23}$ and $3.9 \%{ }^{24}$ reported. Further experience is needed to better define the overall PEG dependency rate with longer follow-up and what the impact of adjuvant radiation and chemoradiation is to this risk, given the level 1 evidence that now supports indications for concurrent chemoradiation. ${ }^{25-27}$

In summary, transoral surgery, such as TORS appears to be reducing the risk of swallowing dysfunction and PEG dependency even with postoperative chemoradiation. This is an important observation as the swallowing dysfunction reported with primary chemoradiation has been shown to affect many qualities of life domains ${ }^{28}$ which is increased with the addition of concurrent chemotherapy $y^{29,30}$ or when treatment requires irradiation of large volumes of the constrictor muscles and laryngopharynx to radiation doses $>50 \mathrm{~Gy}^{31,32}$

Though PEG dependency rates provide arguably a more robust endpoint to evaluate for the risk of late swallowing dysfunction that is clinically meaningful, there are other functional indices valuable. Iseli and colleagues reported the results of the MD Anderson Dysphagia Inventory (MDADI) to evaluate swallowing function at pre-treatment baseline and then at 2 months post-treatment in 54 patients with squamous carcinomas involving multiple sites treated with TORS. ${ }^{33}$ Factors significantly associated with decreased MDADI scores included age $>60(p=0.017)$, T3 and T4 ( $p=0.009)$, laryngeal site $(p=0.017)$, preoperative feeding tube dependence $(p=0.02)$ and postoperative complications $(p=0.035)$. Moore and colleagues assessed swallowing function with the FOSS scale and noted that all of their patients swallowing dysfunction had a base of tongue primary tumors and received adjuvant postoperative chemoradiation. ${ }^{22}$ The two patients in this study who showed abnormal swallowing (FOSS $\geq 3$ ) had T4a base of tongue carcinomas and also presented with a baseline FOSS score of 2 indicating baseline abnormal swallowing function due to the carcinoma. While longer follow-up is needed to better define the clinical limits in which TORS can be safely applied, it is reasonable to expect excellent swallowing function under the newly approved FDA guidelines for using TORS.

A remaining limitation of TORS is the potential for aborting the procedure if adequate exposure to the tumor site is not attenable. Experience to date would suggest that this risk is $<10 \%{ }^{33,34}$ Other factors that may impede adequate TORS exposure are limited neck mobility especially, with limited hyperextension, narrow mandibular arch and retrognathia limit the ability to achieve sufficient transoral exposure. ${ }^{22}$

\section{CURRENT INDICATIONS}

As of December 2009, the Federal Drug Administration (FDA) has approved the use of TORS and the da Vinci ${ }^{\circledR}$ 
surgical system for all T1 and T2 malignancies of the oral cavity, pharynx and larynx and all benign diseases. Therefore, when surgeons utilize these techniques for T3 and $\mathrm{T} 4$ cancers, the patient must be informed that this is off-label use of the robotic technology and should be done utilizing a prospective institutional review board sanctioned protocol. The scope of indications for TORS is beginning to be defined with the greatest body of experience and expertise seen in the management of oropharyngeal squamous cell carcinomas. Other malignant histologies have been treated with TORS but are without sufficient patient numbers, duration of follow-up and independent verification by different institutions to provide confidence in the ability to generalize the use of TORS at this time.

\section{Oropharynx}

Early experiences of transoral robotic surgery has not only demonstrated promising results but also the potential to have a significant impact in the management of head and neck malignancies, especially for resectable oropharyngeal carcinomas. ${ }^{5,8,22,33-35}$ The management of resectable oropharyngeal carcinomas has gravitated in the past 20 years away from a primary surgical approach due to concerns over function loss associated with traditional open transcervical and transmandibular approaches. ${ }^{36}$ This has lead to the development of alternative treatment approaches, including concurrent chemoradiotherapy ${ }^{37}$ and the use of altered fractionated radiotherapy schedules ${ }^{38}$ in combination with concurrent chemotherapy. While these approaches are active and considered established means of treating OP carcinoma, it is now clear that nonconformal radiation techniques can also increase the risk of swallowing complications. ${ }^{29,30,39}$ Even conformal techniques, such as intensity modulated radiotherapy (IMRT) can still be associated with an increased risk of swallowing complications, especially when the constrictor muscles are included in the radiation target volume. $^{32}$ The experiences with TORS at the University of Pennsylvania offer significant promise for both high rates of local and regional disease control rates but also a reduction in the risk of immediate and late swallowing complications seen in chemoradiation approaches.

With a mean follow-up of 26 months, a 2 years actuarial disease-specific survival and disease-free survival of $90 \%$ and $79 \%$ have been observed which appear to be consistent with other early TORS experiences. ${ }^{24}$ A vast majority of relapses (4 of 7) have been distant with only one local and 2 neck relapses observed to date. These results reflect not only an upfront TORS resection but also the pathologic risk stratification guiding adjuvant therapy that in turn may be contributing to the 0\% PEG dependency rate seen at the University of Pennsylvania. ${ }^{21}$ With negative TORS margins and based on neck indications alone, $70 \%$ and $30 \%$ of patients with $\mathrm{N} 1$ and N2b disease respectively did not have any indications for concurrent cisplatin which would otherwise have been administered based on clinical evaluation of the neck disease. TORS also facilitates the use of postoperative doses that are closer to the threshold for injury to the swallowing organs. ${ }^{31,32}$ A key advantage of the transoral surgical approach is the ability to resect the primary site without opening the neck which would otherwise increase the risk of potential tumor seeding ${ }^{40}$ and the volume of the surgical bed that warrants irradiation. Resection also provides greater confidence in the anatomic localization of the primary disease extent with potentially less radiation to the swallowing muscles with the removal of any gross disease extending to the pharyngeal lumen.

As such, a leading hypothesis is that TORS may be further contributing to a reduced risk of late swallowing complications by reducing the volume of the primary tumor bed in turn reducing the radiation dose to the surrounding constrictor muscles even when postoperative chemoradiation is indicated. ${ }^{25-27}$ Consistent though not definitive evidence is the finding that the risk of tube dependence in patients treated with TORS increased with higher T-stage disease. ${ }^{33}$ This does not exclude the possibility that this association may have been due to large T-stages tumors causing increased normal tissue injury as pretreatment tube placement was also a risk factor. In summary, the evidence to date would suggest that TORS for oropharyngeal carcinomas is associated with high rates of local disease control but may also be a strategy to reduce the risk of late swallowing complications as measured by PEG dependency rates. The tumor-related indications for oropharyngeal TORS resection at the University of Pennsylvania include previously untreated biopsy proven squamous cell carcinoma of the oropharynx which was American Joint Committee on Cancer (AJCC) stage III, IVA and IVB including AJCC T1, T2, T3 and T4a cancers. The tumor-related contraindications for TORS resection include: (1) Stage IVC with the exception of a curable distant metastasis, (2) unresectability of the involved lymph nodes, 
(3) all AJCC TNM T4a with the exception of unilateral deep/extrinsic muscle of the tongue or tumor-related trismus, (4) all AJCC TNM T4b and (5) any AJCC T stage including the invasion of the deep tissues lateral to the constrictor muscles or posterior invasion of the prevertebral fascia. This lateral and deep invasion may be noted radiologically but also must be confirmed as fixation laterally or posteriorly by palpation. Nodal unresectability is defined as carotid artery encasement, deep neck structure involvement resulting in the prediction that the node could not be grossly resected and skin invasion with dermal metastasis. Nontumor related contraindications include: (1) benign causes of trismus or other anatomical findings which preclude transoral access, (2) retropharyngeal internal carotid artery in which the artery is located directly behind the tonsillar fossa; this is a contraindication for TORS radical tonsillectomy but not for resection of other oropharyngeal sites, (3) medical contraindications for either general anesthesia or transoral surgery in which an open wound heals by secondary intention (i.e. need for chronic anticoagulation).

\section{Larynx/Hypopharynx}

The feasibility and safety for TORS in the treatment of laryngeal carcinomas was first demonstrated by Weinstein and O'Malley in the canine model,,6 including endolaryngeal resection ${ }^{4}$ and in human patients with supraglottic carcinomas. ${ }^{7}$ Other institutions have also confirmed the feasibility and safety of this technique with cadaveric studies $^{41}$ and in humans with laryngeal and hypopharyngeal carcinomas. ${ }^{33-35}$ These investigators concluded that the robotic arms readily facilitated excellent surgical exposure with magnified 3D images and threedimensional resection due to the full $360^{\circ}$ rotational and multiplanar resection abilities. In particular, complex anatomic structures, such as the paraglottic space could be effectively visualized and dissected. In contrast, line of sight limitations when conventional transoral approaches using the laryngoscope and microscope to resect such complex structures limit not only the safety but also the adequacy of the surgical resection. No surgical complications or mortality were reported. ${ }^{7,35}$ While the long-term oncologic outcomes with TORS for laryngeal and hypopharyngeal carcinomas has not been reported, this promising technique warrants further evaluation as it may offer further improved organ preserving function due to the preserved pharyngeal sensation with the transoral vs the transcervical approach.
However, caution is warranted given the suggestion that TORS to this site may be associated with an increased risk of postoperative dysphagia. ${ }^{33,34}$

The present indications at the University of Pennsylvania for TORS supraglottic partial laryngectomy include selected T1, T2 and T3 cancers of the supraglottis. The present contraindications are consistent with the contraindications of open supraglottic partial laryngectomy, ${ }^{42}$ including (1) invasion of the cricoids and/or thyroid cartilage, (2) bilateral mucosal invasion of the arytenoid cartilage, (3) invasion of the posterior and anterior commissure, (4) fixation of the arytenoids and the true vocal cord, (5) involvement of the base of the tongue closer than $1 \mathrm{~cm}$ to the circumvallate papillae, (6) imparted motion of the tongue base as assessed by indirect laryngoscopy and (7) invasion of the floor of the mouth in vallecular carcinoma.

\section{Skull Base/Nasopharynx}

O’Malley and Weinstein have reported cadaveric studies and human feasibility studies of a TORS approach to the parapharyngeal space and infratemporal fossa ${ }^{3}$ and a modified TORS approach for successful access and dissection at the anterior and middle skull base termed CTORS. ${ }^{9}$ Of note C-TORS has only been done in the preclinical setting on cadavers and is not at this time recommended for use in patients. Access to the anterior skull base is limited by the ability to place the transoral robotic instruments above the hard and soft palate as required. To circumvent this limitation, a novel combined transoral and transnasal approach has been developed by O’Malley, Weinstein, Lee, and Newman and is under investigation in both preclinical and human clinical trials.

The use of TORS for well-defined parapharyngeal space masses has also been investigated in humans. ${ }^{3}$ While the role of TORS in the management of parapharyngeal masses continues to be the subject of investigation, it is clear that for benign tumors, this technique appears to be feasible, safe and effective. ${ }^{3}$ For malignant tumors, the use of TORS must be viewed cautiously and should be considered a relative contraindication at this time given the high rates of local relapses (36-75\%) reported even with open techniques. ${ }^{43-45}$ As preoperative fine needle aspiration biopsies are often equivocal, prudence would favor an open approach under these circumstances. Additional contraindications include osseous skull base and carotid involvement. 
This technique also holds promise to safely expand the indications of surgical resection for diseases at the nasopharynx reducing the morbidity associated with traditional open approaches ${ }^{46}$ and improving on recent endoscopic techniques that have been successfully described for the salvage of recurrent T1-2a nasopharyngeal carcinomas. ${ }^{47}$ The potential to improve upon these techniques with the advantages of the TORS approach in the management of recurrent nasopharyngeal carcinomas was recent described by Wei and Ho. ${ }^{48}$ Ongoing studies continue to fully evaluate the spectrum of potential indications and limitations from this technique at the skull base and preclude any generalized indications at this time.

\section{Other Benign Diseases}

Benign diseases are in principle well suited for TORS, especially for well-defined lesions. In their prospective TORS human clinical trials of over 400 patients to date, Weinstein and O'Malley tested the feasibility of TORS for various benign lesions and tumors, including lingual tonsillar and base of tongue masses and hypertrophy (unpublished data). In the course of these procedures, there were patients with sleep disturbances who communicated a significant improvement in their sleep after TORS for their base of tongue benign lesions. The application of TORS for BOT lesions established the precedent for investigating TORS for sleep apnea. As an example, Vicini and colleagues have recently reported on early outcome data with TORS for the management of patients with obstructive sleep apneahypoapnea syndrome primarily related to hypertrophy of the tongue base. ${ }^{49}$ Both feasibility and successful reduction in the apnea-hypopnea index was demonstrated in patients at a minimum of 3 months follow-up.

\section{SUMMARY}

In summary, TORS and the application of robotic technology in general offers a significantly less morbid approach and may improve the ability to achieve negative margin surgery in the management of oropharyngeal carcinomas. The favorable oncologic outcomes with TORS to date are at least comparable to standard chemotherapy and radiation treatment. TORS has demonstrated comparable, if not significantly, lower rates of late swallowing complications and dysfunction as measured by feeding tube dependence rates. In addition to the treatment of OP carcinomas, TORS has shown early feasibility and success for benign and selected malignant diseases for the larynx, hypopharynx, parapharyngeal space and infratemporal fossa with malignant lesions carefully selected based on the depth of deep tumor infiltration. TORS may also be a promising strategy for the surgical salvage of early recurrent nasopharyngeal carcinomas. While promising, there is a need for larger patient experiences and longerfollow-up before the benefit of TORS across the numerous applications can be firmly substantiated.

\section{REFERENCES}

1. Hockstein NG, O’Malley BW (Jr). Weinstein GS. Assessment of intraoperative safety in transoral robotic surgery. The Laryngoscope. Feb 2006;116(2):165-68.

2. Hockstein NG, Weinstein GS, O’Malley Jr BW. Maintenance of hemostasis in transoral robotic surgery. ORL; journal for otorhinolaryngology and its related specialties. 2005;67(4):220-24.

3. O’Malley BW (Jr), Weinstein GS. Robotic skull base surgery: Preclinical investigations to human clinical application. Archives of otolaryngology-head and neck surgery. Dec 2007;133(12):1215-19.

4. O’Malley BW (Jr), Weinstein GS, Hockstein NG. Transoral robotic surgery (TORS): Glottic microsurgery in a canine model. J Voice Jun 2006;20(2):263-68.

5. O’Malley BW (Jr), Weinstein GS, Snyder W, Hockstein NG. Transoral robotic surgery (TORS) for base of tongue neoplasms. The Laryngoscope. Aug 2006;116(8):1465-72.

6. Weinstein GS, O’Malley BW (Jr), Hockstein NG. Transoral robotic surgery: Supraglottic laryngectomy in a canine model. The Laryngoscope. Jul 2005;115(7):1315-19.

7. Weinstein GS, O’Malley BW (Jr), Snyder W, Hockstein NG. Transoral robotic surgery: Supraglottic partial laryngectomy. The Annals of otology, rhinology, and laryngology. Jan 2007;116(1):19-23.

8. Weinstein GS, O’Malley BW (Jr), Snyder W, Sherman E, Quon H. Transoral robotic surgery: Radical tonsillectomy. Archives of otolaryngology-head and neck surgery. Dec 2007;133(12):1220-26.

9. O’Malley BW (Jr), Weinstein GS. Robotic anterior and midline skull base surgery: Preclinical investigations. International journal of radiation oncology, biology, physics. 2007;69(2 Suppl):S125-28.

10. Laccourreye O, Hans S, Menard M, Garcia D, Brasnu D, Holsinger FC. Transoral lateral oropharyngectomy for squamous cell carcinoma of the tonsillar region: II. An analysis of the incidence, related variables, and consequences of local recurrence. Archives of otolaryngology — head and neck surgery. Jul 2005;131(7):592-99.

11. Donias HW, Karamanoukian HL, D’Ancona G, Hoover EL. Minimally invasive mitral valve surgery: From Port Access to fully robotic-assisted surgery. Angiology. Jan 2003;54(1): 93-101.

12. Steiner W, Fierek O, Ambrosch P, Hommerich CP, Kron M. Transoral laser microsurgery for squamous cell carcinoma of the base of the tongue. Archives of otolaryngology — head and neck surgery. Jan 2003;129(1):36-43. 
13. Grant DG, Salassa JR, Hinni ML, Pearson BW, Perry WC. Carcinoma of the tongue base treated by transoral laser microsurgery, part one: Untreated tumors, a prospective analysis of oncologic and functional outcomes. The Laryngoscope Dec 2006;116(12):2150-55.

14. Grant DG, Hinni ML, Salassa JR, Perry WC, Hayden RE, Casler JD. Oropharyngeal Cancer: A Case for Single Modality Treatment with Transoral Laser Microsurgery. Archives of otolaryngology - head and neck surgery. 1 December, 2009;135(12):1225-30.

15. Eckel HE, Volling P, Pototschnig C, Zorowka P, Thumfart W. Transoral laser resection with staged discontinuous neck dissection for oral cavity and oropharynx squamous cell carcinoma. The Laryngoscope 1995;105(1):53-60.

16. Rich JT, Milov S, Lewis JS (Jr), Thorstad WL, Adkins DR, Haughey BH. Transoral laser microsurgery (TLM) +/- adjuvant therapy for advanced stage oropharyngeal cancer: Outcomes and prognostic factors. The Laryngoscope. Sep 2009;119(9):1709-19.

17. Weinstein GS, O’Malley BW (Jr), Snyder W, Sherman E, Quon H. Transoral Robotic Surgery: Radical Tonsillectomy. Archives of otolaryngology-head and neck surgery. 1 December, 2007;133(12):1220-26.

18. Ang KK, Peters LJ, Weber RS, Morrison WH, Frankenthaler RA, Garden AS, et al. Postoperative radiotherapy for cutaneous melanoma of the head and neck region. International journal of radiation oncology, biology, physics. Nov 15, 1994;30(4):795-98.

19. Haughey BH, Taylor SM, Fuller D. Fasciocutaneous flap reconstruction of the tongue and floor of mouth: Outcomes and techniques. Archives of otolaryngology — head and Neck Surgery Dec 2002;128(12):1388-95.

20. Salassa JR. A functional outcome swallowing scale for staging oropharyngeal dysphagia. Digestive diseases (Basel, Switzerland). 1999;17(4):230-34.

21. Dosoretz A, Dutta P, Alonso-Basanta M, BarAd V, Both S, Lin A, et al. Long-term Percutaneous Gastrostomy Tube Dependence Rates in Patients Treated with Intensity-Modulated Radiotherapy for Oropharyngeal Cancer: The University of Pennsylvania Experience. ASTRO Multidisciplinary Head and Neck Cancer Symposium; 2009; Chandler, Arizona; 2009.

22. Moore EJ, Olsen KD, Kasperbauer JL. Transoral robotic surgery for oropharyngeal squamous cell carcinoma: A prospective study of feasibility and functional outcomes. The Laryngoscope. Nov 2009;119(11):2156-64.

23. Holsinger FC, McWhorter AJ, Menard M, Garcia D, Laccourreye O. Transoral lateral oropharyngectomy for squamous cell carcinoma of the tonsillar region: I. Technique, complications, and functional results. Archives of otolaryngology—head and neck surgery. Jul 2005;131(7):58391.

24. Moore EJ, Henstrom DK, Olsen KD, Kasperbauer JL, McGree ME. Transoral resection of tonsillar squamous cell carcinoma. The Laryngoscope. Mar 2009;119(3):508-15.

25. Bernier J, Domenge C, Ozsahin M, Matuszewska K, Lefebvre JL, Greiner RH, et al. Postoperative irradiation with or without concomitant chemotherapy for locally advanced head and neck cancer. The New England journal of medicine. 6 May, 2004;350(19):1945-52.

26. Cooper JS, Pajak TF, Forastiere AA, Jacobs J, Campbell BH, Saxman SB, et al. Postoperative concurrent radiotherapy and chemotherapy for high-risk squamous-cell carcinoma of the head and neck. The New England journal of medicine. 6 May, 2004;350(19):1937-44.

27. Bernier J, Cooper JS, Pajak TF, van Glabbeke M, Bourhis J, Forastiere A, et al. Defining risk levels in locally advanced head and neck cancers: A comparative analysis of concurrent postoperative radiation plus chemotherapy trials of the EORTC (\#22931) and RTOG (\# 9501). Head and Neck Oct 2005;27(10):843-50.

28. Langendijk JA, Doornaert P, Verdonck-de Leeuw IM, Leemans CR, Aaronson NK, Slotman BJ. Impact of Late TreatmentRelated Toxicity on Quality of Life Among Patients With Head and Neck Cancer Treated With Radiotherapy. J Clin Oncol 1 August, 2008;26(22):3770-76.

29. Langendijk JA, Doornaert P, Rietveld DH, Verdonck-de Leeuw IM, Leemans CR, Slotman BJ. A predictive model for swallowing dysfunction after curative radiotherapy in head and neck cancer. Radiother Oncol. Feb 2009;90(2):189-95.

30. Caudell JJ, Schaner PE, Meredith RF, Locher JL, Nabell LM, Carroll WR, et al. Factors Associated with Long-term Dysphagia After Definitive Radiotherapy for Locally Advanced Head-andNeck Cancer. International journal of radiation oncology, biology, physics. 15 July, 2008.

31. Feng FY, Kim HM, Lyden TH, Haxer MJ, Feng M, Worden FP, et al. Intensity-modulated radiotherapy of head and neck cancer aiming to reduce dysphagia: Early dose-effect relationships for the swallowing structures. International journal of radiation oncology, biology, physics. 1 Aug 2007;68(5):1289-98.

32. Levendag PC, Teguh DN, Voet P, van der Est H, Noever I, de Kruijf WJ, et al. Dysphagia disorders in patients with cancer of the oropharynx are significantly affected by the radiation therapy dose to the superior and middle constrictor muscle: A doseeffect relationship. Radiother Oncol Oct 2007;85(1):64-73.

33. Iseli TA, Kulbersh BD, Iseli CE, Carroll WR, Rosenthal EL, Magnuson JS. Functional outcomes after transoral robotic surgery for head and neck cancer. Otolaryngol Head Neck Surg. Aug 2009;141(2):166-71.

34. Genden EM, Desai S, Sung CK. Transoral robotic surgery for the management of head and neck cancer: A preliminary experience. Head \& neck. 2009 Mar;31(3):283-89.

35. Park YM, Lee WJ, Lee JG, Lee WS, Choi EC, Chung SM, et al. Transoral robotic surgery (TORS) in laryngeal and hypopharyngeal cancer. Journal of laparoendoscopic \& advanced surgical techniques. 2009 Jun;19(3):361-68.

36. Gourin CG, Johnson JT. Surgical treatment of squamous cell carcinoma of the base of tongue. Head \& neck. 2001 Aug;23(8):653-60.

37. Pignon J-P, le Maître A, Bourhis J. Meta-Analyses of Chemotherapy in Head and Neck Cancer (MACH-NC): An Update. International Journal of Radiation Oncology* Biology* Physics. 2007;69(2, Supplement 1):S112-S4.

38. Bourhis J, Overgaard J, Audry H, Ang KK, Saunders M, Bernier J, et al. Hyperfractionated or accelerated radiotherapy in head and neck cancer: A meta-analysis. Lancet. 2006 Sep 2;368(9538):843-54.

39. Machtay M, Moughan J, Trotti A, Garden AS, Weber RS, Cooper JS, et al. Factors associated with severe late toxicity after concurrent chemoradiation for locally advanced head and neck cancer: An RTOG analysis. J Clin Oncol. 2008 Jul 20;26(21):3582-89. 
40. Fletcher GH, Evers WT. Radiotherapeutic management of surgical recurrences and postoperative residuals in tumors of the head and neck. Radiology. Apr 1970;95(1):185-88.

41. Solares CA, Strome M. Transoral robot-assisted $\mathrm{CO}_{2}$ laser supraglottic laryngectomy: Experimental and clinical data. The Laryngoscope May 2007;117(5):817-20.

42. Weinstein GS, Laccourreye O, Brasnu D, Laccourreye H (Eds). Supraglottic Laryngectomy. Organ Preservation Surgery for Laryngeal Cancer. San Diego, California: Singular Publishing Group; 2000.

43. Hughes KV (3rd), Olsen KD, McCaffrey TV. Parapharyngeal space neoplasms. Head and neck. Mar-Apr 1995;17(2): 124-30.

44. Carrau RL, Myers EN, Johnson JT. Management of tumors arising in the parapharyngeal space. The Laryngoscope. Jun 1990;100(6):583-89.

45. Malone JP, Agrawal A, Schuller DE. Safety and efficacy of transcervical resection of parapharyngeal space neoplasms. The
Annals of otology, rhinology, and laryngology. Dec 2001;110(12):1093-98.

46. Wei WI, Lam KH, Sham JS. New approach to the nasopharynx: The maxillary swing approach. Head \& neck. May-Jun 1991;13(3):200-07.

47. Chen MK, Lai JC, Chang CC, Liu MT. Minimally invasive endoscopic nasopharyngectomy in the treatment of recurrent T1-2a nasopharyngeal carcinoma. The Laryngoscope. 2007 May;117(5):894-96.

48. Wei W, Ho W. Transoral robotic resection of recurrent nasopharyngeal carcinoma. World Robotic Symposium, Orlando, Florida; 2010.

49. Vicini C, Dallan I, Canzi P, Frassineti S, La Pietra MG, Montevecchi F. Transoral Robotic Tongue Base Resection in Obstructive Sleep Apnoea-Hypopnoea Syndrome: A Preliminary Report. ORL; journal for otorhinolaryngology and its related specialties. Feb 18;72(1):22-27. 